\title{
Cochlear implants: Insertion assessment by computed tomography ${ }^{\text {in }}$
}

\author{
Ana Luiza Menegatti Pavan ${ }^{\mathrm{a}}$, Allan Felipe Fattori Alves ${ }^{\mathrm{a}}$, Guilherme Giacomini ${ }^{\mathrm{a}}$, \\ João Maurício Carrasco Altemani ${ }^{\mathrm{b}}$, Arthur Menino Castilho ${ }^{\mathrm{b}}$, Raquel Andrade Lauria ${ }^{\mathrm{b}}$, \\ Vagner Antonio Rodrigues da Silva ${ }^{\mathrm{b}}$, Alexandre Caixeta Guimarães ${ }^{\mathrm{b}}$, Diana Rodrigues de Pina ${ }^{\mathrm{c}, *}$ \\ ${ }^{a}$ Instituto de Biociências de Botucatu, Universidade Estadual Paulista (IBB-UNESP), Botucatu, São Paulo, Brazil \\ ${ }^{\mathrm{b}}$ Hospital de Clínicas, Universidade Estadual de Campinas (HC-UNICAMP), Campinas, São Paulo, Brazil \\ ${ }^{\mathrm{c}}$ Faculdade de Medicina de Botucatu, Universidade Estadual Paulista (FMB-UNESP), Botucatu, São Paulo, Brazil
}

\section{A R T I C L E I N F O}

\section{Keywords:}

Cochlear implants

Computed tomography

Insertion measurements

Image reconstruction

\begin{abstract}
A B S T R A C T
Background and objectives: Imaging exams play a key role in cochlear implants with regard to both planning implantation before surgery and quality control after surgery. The ability to visualize the three-dimensional location of implanted electrodes is useful in clinical routines for assessing patient outcome. The aim of this study was to evaluate linear and angular insertion depth measurements of cochlear implants based on conventional computed tomography.

Methods: Tools for linear and angular measurements of cochlear implants were used in computed tomography exams. The tools realized the insertion measurements in an image reconstruction of the CIs, based on image processing techniques. We comprehensively characterized two cochlear implant models while obviating possible changes that can be caused by different cochlea sizes by using the same human temporal bones to evaluate the implant models.

Results: The tools used herein were able to differentiate the insertion measurements between two cochlear implant models widely used in clinical practice. We observed significant differences between both insertion measurements because of their different design and construction characteristics $(p=0.004$ and 0.003 for linear and angular measurements, respectively; $t$-test). The presented methodology showed to be a good tool to calculate insertion depth measurements, since it is easy to perform, produces high-resolution images, and is able to depict all the landmarks, thus enabling measurement of the angular and linear insertion depth of the most apical electrode contacts.

Conclusion: The present study demonstrates practical and useful tools for evaluating cochlear implant electrodes in clinical practice. Further studies should measure preoperative and postoperative benefits in terms of speech recognition and evaluate the preservation of residual hearing in the implanted ear. Such studies can also determine correlations between surgical factors, electrode positions, and performance. In addition to refined surgical techniques, the precise evaluation of cochlear length and correct choice of cochlear implant characteristics can play an important role in postoperative outcomes.
\end{abstract}

\section{Introduction}

Cochlear implants (CIs) functionally restore hearing in individuals with profound hearing impairment [1-4]. An array of electrodes is introduced into the cochlea, and electrical pulses are applied across the array to stimulate residual populations of dendritic and spiral ganglion nerve bodies. Most individuals are able to obtain 40-80\% correct postoperative word recognition with their CI compared with zero or only modest scores preoperatively [5]. Although the implantation procedure is safe and reliable, complications occur in approximately $16 \%$ of patients [6]. Following cochlear implantation, there is the possibility that residual hearing can be lost because of the surgical procedure itself or progression of the underlying pathology $[7,8]$. A greater focus has been placed on minimizing insertion trauma and preserving preexisting hearing $[9,10]$. For electroacoustic stimulation to be the most effective, the patient's residual hearing should be preserved during CI surgery [4,7,10-12]. Thus, selecting the optimal insertion depth should be patient-specific and based on the corresponding

\footnotetext{
Conflict of Interest: The authors declare that they have no conflict of interest.

* Corresponding author at: Faculdade de Medicina de Botucatu, Departamento de Doenças Tropicais e Diagnóstico por Imagem, Universidade Estadual Paulista—UNESP, Distrito de Rubiao Junior S/N, Botucatu 18618-000, São Paulo, Brazil.

E-mail address: drpina@fmb.unesp.br (D.R. de Pina).
} 
residual hearing $[9,13]$.

Electroacoustic stimulation is an excellent option for people who have residual hearing at low frequencies but not at high frequencies and achieve insufficient benefits from hearing aids [4,7,10-12,14]. The auditory performance of CIs presents substantial interindividual variability, partly because of the quality of the interface between the electrode and auditory nerve endings [15]. The size of human cochleae also varies considerably $[1,9,13]$. Although the presence of interindividual variations in anatomy and the goal of preserving residual hearing, it is still not known if these variations of the size of the cochlea duct reflect in differing ability to stimulate the spiral ganglion. $\mathrm{CI}$ electrodes could be adapted to patients individually by objective measurements of individual variations of the cochlea [16]. Growing interest has been seen in precisely documenting the position of individual electrode contacts relative to cochlear structures, the insertion depth of the electrode array, and potential insertion trauma [17]. Some authors believe that individualized CI electrode arrays and adjusted insertion procedures that take into account the individual cochlear length are relevant for the hearing preservation [16]. The development of a tool to measure the insertion depth would be valuable in clinical routines [13].

Imaging exams play a key role in CIs with regard to both planning the implantation procedure and, in sometimes, for quality control (i.e., accurately confirming the electrode array position in the cochlear scala) after surgery, mainly in cases where results from neural response telemetry (NRT) are inaccurate to check placement of the electrode [1,2]. Detailed preoperative evaluations of intracochlear structures help surgeons plan the surgery, select the electrode model, design the surgical procedure, and avoid possible intraoperative complications. Postoperative evaluations could help surgeons determine whether the electrode array has migrated between the scalae, which is a direct measure of potential intracochlear trauma and can have a negative impact on the preservation of hearing. Postoperative evaluations are also used to identify possible electrode kinks, buckling, or tip folding and to take necessary countermeasures by switching off the contacts in that region to improve the performance of the CI [1,2]. Imaging exams are commonly used to evaluate these important interindividual cochlear differences and have the potential to guide cochlear implantation [1].

Electrode insertion measurements (i.e., the length of insertion and insertion angle) are relevant for estimating the percentage of cochlear coverage with electrical stimulation. This measure can be used as an indicator of the performance of the profoundly deaf patient postoperatively $[1,12,18]$. Studies have shown that specifying the electrode position in terms of angle might be more useful than specifying the length. Because cochlea differ in diameter, equal insertion lengths result in different insertion angles and thus different frequency characteristics. Furthermore, the insertion angle depends on the intracochlear trajectory of the electrode [12].

The ability to visualize cochlear anatomy and the three-dimensional (3D) location of implanted electrodes relative to landmarks in the ear is useful in clinical routines for assessing patient outcome [19]. Conventional computed tomography (CT) is particularly useful because it provides 3D positional information, offers excellent contrast for different types of tissue, and can be utilized even with the implant device in plane $[2,16,19]$. High-resolution CT scans and novel postprocessing algorithms, such as multiplanar reconstruction (MPR) images, provide valuable information about the electrode array position [15,19]. However, specific relationships between array positions, electrophysiological parameters, and auditory performance remain to be established. This issue is critical because it can directly influence the array design and implantation technique [15].

The aim of the present study was to evaluate linear insertion depth (LID) and insertion depth angle (IDA) using two tools based on CT exams. We comprehensively characterized two CI models by evaluating linear and angular depth. The same human temporal bones were used to evaluate both implant models while keeping the cochlea size constant.

\section{Materials and methods}

\subsection{Compliance with ethical standards}

All of the experiments conformed with the requirements of the Ethics Committee of Universidade Estadual de Campinas for the ethical use of human material, under protocol number CAAE41305414.0.0000.5404

\subsection{Electrodes}

The modiolar research array (MRA; Cochlear Implant A; Cochlear ${ }^{\circledR}$, Australia) is a thin prototype precurved array with an apical diameter of $0.3 \mathrm{~mm}$ and basal diameter of $0.5 \mathrm{~mm}$. This device has 22 half-band platinum electrodes that are surrounded by an external polymer sheath ( $0.65 \mathrm{~mm}$ diameter), which is removed after full insertion of the array. The removal of the silicone sheath reduces the electrode volume and results in a flexible electrode with dimensions that are equivalent to lateral wall electrodes that are designed to preserve residual hearing. The surrounding silicone has a soft tip to prevent trauma and a stopper that limits the insertion depth of the sheath for an Advance Off-Stylet style of insertion. The MRA is $17 \mathrm{~mm}$ long with an approximate angular insertion depth of $390-450^{\circ}$ [20].

The Contour Advance device (CA; Cochlear Implant B; Cochlear ${ }^{\circledast}$, Australia) also contains 22 half-banded electrodes that are designed to face the modiolar side. The precurved electrode array diameter tapers from $0.8 \mathrm{~mm}$ at the basal end to $0.5 \mathrm{~mm}$ at the tip, with platinum electrodes embedded along the initial $15.5 \mathrm{~mm}$ of a silicone carrier. A malleable platinum stylet is placed into a lumen in the silicone carrier to hold the electrode straight for insertion. After removing the stylet, the electrode array assumes its curved perimodiolar shape. A mark at $22 \mathrm{~mm}$ in the silicone carrier guides the depth of insertion of the electrode array [21].

\subsection{Temporal bone}

Five formaldehyde-preserved human temporal bones were implanted with these electrodes in the Hospital de Clínicas of the Universidade Estadual de Campinas (HC-UNICAMP) through the round window membrane (RWM) without surgical problems or complications. The round window niche was exposed by posterior tympanotomy, most of the anterior bony overhang of the round window niche was removed and an extended round window technique was performed. A transverse incision was made across the RWM, and a defined electrode was carefully inserted into the scala tympani through the incised RWM.

The human temporal bones were first implanted with Cochlear Implant A electrodes and then imaged by CT. These electrodes were then removed, and Cochlear Implant B electrodes were implanted in the same temporal bones and imaged by CT. Therefore, our evaluation excluded possible variations that are related to cochlea size. These procedures were carefully performed to preserve the implanted cochlea.

\subsection{Computed tomography}

The CT scans were acquired using a Toshiba Aquilion 64 (Toshiba America Medical Systems, Tustin, CA, USA) with the following parameters: $0.22 \pm 0.05 \times 0.22 \pm 0.05$ pixel size, $512 \times 512$ pixel matrix, and $0.50 \mathrm{~mm}$ slice width. Postoperative CT images were reconstructed to provide a cross-sectional view of the implanted electrode, parallel to the basal turn of the cochlea and perpendicular to the modiolus, corresponding to the plane of the electrode array [17]. Three-dimensional reconstruction of the CIs was performed using itkSNAP software. These reconstructions allowed measurements of the insertion depth $[1,9,13,22]$. 


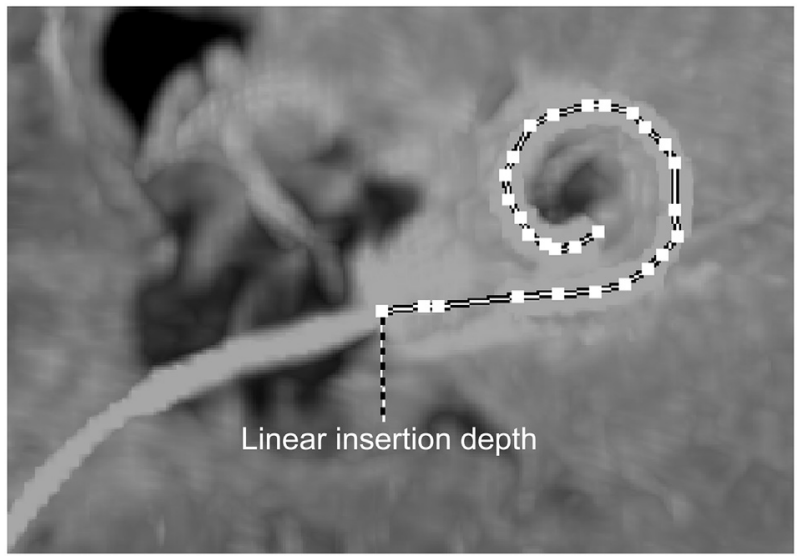

Fig. 1. Example of linear insertion depth in a multiplanar reconstruction of the computed tomography.

\subsection{Measurements}

Expert radiologists with substantial experience in identifying both normal and abnormal cranial structures supervised the CT exams, manipulated the image projection, and made the measurements.

\subsubsection{Linear insertion depth}

To determine the linear insertion depth (LID), the distance between the round window and apical end of the array electrode was measured (in millimeters) using MPR images. This measurement was made carefully tracing the implant contour using the linear measurement function tool of the CT scanner workstation. Fig. 1 shows an example of the LID measurement $[1,13]$.

\subsubsection{Insertion depth angle}

The insertion depth angle (IDA) was measured in a 3D reconstruction of the CIs using itk-SNAP software (Fig. 2A). The reconstruction is made using image processing techniques. In the first step, the original image is thresholded to remove biological structures, highlighting the array electrode regions, which are the regions of interest (ROIs). Then, the user chooses seed points in the ROIs. These seed points are used in an active contour technique, which segments all the array electrode areas present in the slice. The 3D reconstruction is obtained from the ROIs segmentation of all slices of CT exams. The same procedure is realized to obtain the landmark for the round window (gray dot in Fig. 2A).

Fig. 2B represents the IDA. The center of the angle measurement was defined as the center of the modiolus. As a reference, the $0^{\circ}$ angle

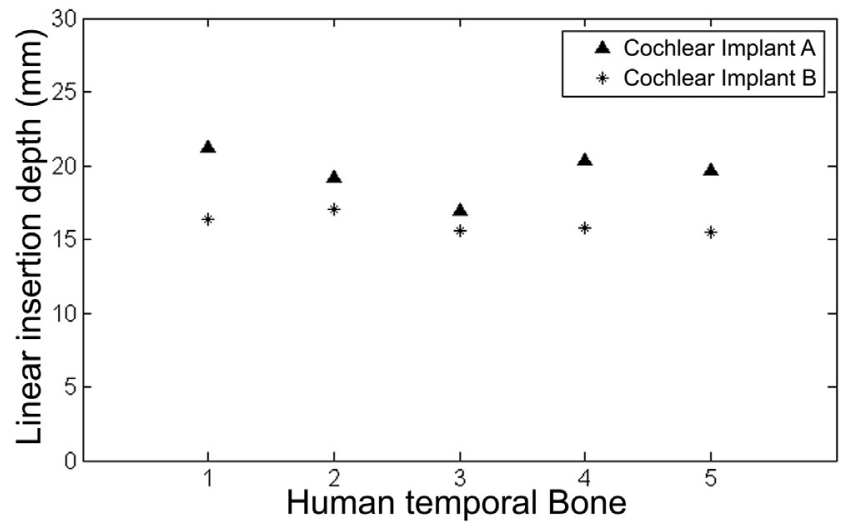

Fig. 3. Linear insertion depth of electrode arrays for the five human temporal bones. Triangles (Cochlear Implant A) and asterisks (Cochlear Implant B) indicate the linear insertion of the most apical electrode, with the round window as a reference.

(white line) coincides with the center of the round window (gray dot). The IDA is the angle between the $0^{\circ}$ reference angle and the end of the CI (black dashed line) $[1,12,13]$.

\subsection{Statistical analysis}

To compare the linear and angular measurements of Cochlear Implants A and B, an independent $t$-test was performed using MiniTab 14 software. Values of $p<0.05$ were considered statistically significant.

\section{Results}

Figs. 3 and 4 show the LID and IDA for the CI surgeries, respectively.

Table 1 presents comparisons of the insertion depth (linear and angular) between Cochlear Implants A and B.

\section{Discussion}

The study evaluated insertion depth measurements (LID and IDA) by conventional CT exam using two practical tools. These measurements may be used to compare different electrode types, to assess the placement of electrodes postoperatively or to compare different insertion techniques [23]. In this study, two CI electrodes (Cochlear Implants A and B) were implanted in the same human temporal bones to exclude possible variations related to cochlea size.

Literature shows that imaging evaluation of postoperative CI
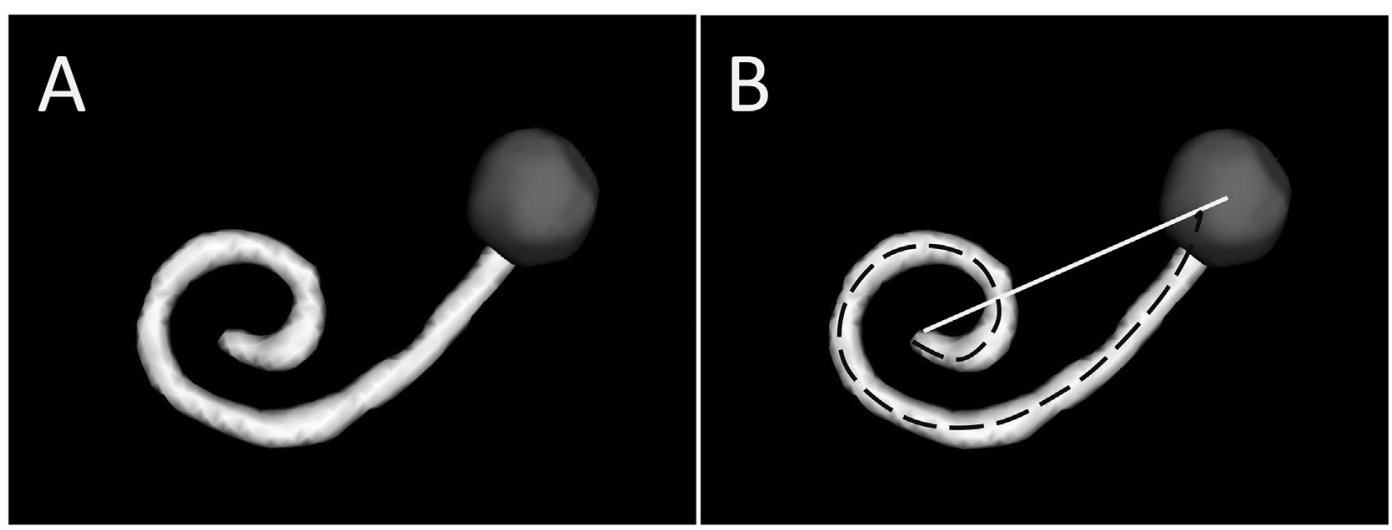

Fig. 2. (A) Example of three-dimensional reconstruction of cochlear implant based on computed tomography. (B) Example of insertion depth angle. The center of the angle measurement coincides with the center of the modiolus. The gray dot represents the round window ( $0^{\circ}$ reference angle). The IDA is represented by a black dashed line. 


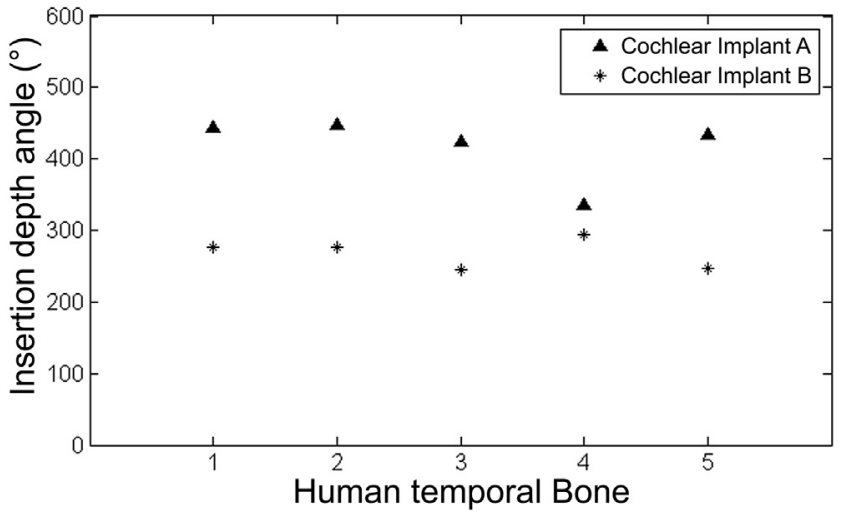

Fig. 4. Insertion depth angle of electrode arrays for the five human temporal bones. Triangles (Cochlear Implant A) and asterisks (Cochlear Implant B) indicate the angular insertion of the most apical electrode, with the round window as a reference $\left(0^{\circ}\right.$ angle).

Table 1

Comparison of insertion depth (linear and angular) between Cochlear Implants $\mathrm{A}$ and B. The data are expressed as mean \pm standard deviation. ${ }^{*} p<0.05$, significant differences between groups (Student's $t$-test).

\begin{tabular}{llll}
\hline & Cochlear implant A & Cochlear implant B & $p$ \\
\hline Linear insertion depth (mm) & $19.5 \pm 1.6$ & $16.1 \pm 0.6$ & $0.004^{*}$ \\
Insertion depth angle $\left(^{\circ}\right)$ & $416.6 \pm 46.8$ & $267.9 \pm 20.9$ & $0.003^{*}$ \\
\hline
\end{tabular}

placement was initially performed with plain radiographs in the Stenvers projection [24]. Radiographs are, in many cases, adequate to acquire immediate clinical information [23]. However, methods that are based only on two-dimensional data critically depend of the orientation of the patient's head during imaging [12]. Therefore, nonstandardized acquisition of the skull radiograph may be a disadvantage when measuring angular depth of insertion and position of electrode [23].

Conventional CT replaced planar radiographs method and has been the reference standard imaging technique for assessing the electrode array location relative to intracochlear scalae, the electrode-modiolar interval, and the proximity of the electrode to the fallopian canal. CT offers the ability to provide 3D positional information and excellent contrast for different tissue types [24]. Therefore, 3D images reconstructed from CT scans results in higher accuracy because reference landmarks may be defined more precisely [12]. Furthermore, 3D images do not rely on the orientation of the patient's head during the scanning procedure. Nonetheless, the 3D images require the definition of reference points, but more landmarks can be selected compared with two-dimensional images [12]. One caveat of CT, however, is the possible presence of metallic artifacts that can limit visualization $[12,24,25]$. This limitation did not affect the insertion measurements carried out by the assessed methodology herein, since the reconstructed CT exam accurately shows the 3D position of all used landmarks.

Other 3D image modalities (cone beam CT, micro CT and flat-panel CT) carry several advantages over conventional CT, such as to create less metallic artifacts and to offer higher spatial resolution and accuracy [13,24,26-29]. However, these modalities have their use limited by cost and restricted accessibility, impairing the applicability in clinical practice. Thus, it is of great interest to use a methodology applicable in centers where there are only conventional CT available.

Figs. 3 and 4 show comparisons of the LID and IDA for the same human temporal bone. The IDA can vary considerably, with ranges of $113^{\circ}$ and $50^{\circ}$ for Cochlear Implants A and B, respectively. Such variations are caused by different dimensions of the human cochleae or different intracochlear trajectories of the arrays with regard to scale dislocation or the distance to the modiolus. Depending on the size of the cochlea, a fixed electrode length can result in different insertion angles. Therefore, length measurements may be essential for anatomical reasons and hearing preservation.

The well-known benefits of CIs have been established for electrodes that have been designed to be inserted to a depth that exceeds $360^{\circ}$. Some studies reported that deeper electrode insertion is associated with better postoperative speech recognition. Recent studies have postulated that preserving the delicate structure of the inner ear must be a goal of all CI surgeries with or without significant residual hearing prior to implantation [5]. Several shorter electrodes have been designed to avoid insertion trauma [5]. O'Connell et al. 2016 concluded that intracochlear electrode position has direct influence in speech recognition [30]. Therefore, new studies that evaluate different cochlear implantation measurements are useful for practical routines, which may directly improve postoperative patient outcomes [16]. The variability of the insertion depth should be taken into account when individually developing the adapted length of electrode arrays for patients with residual hearing [12].

The methodology presented herein was able to differentiate the insertion measurements between two CI models widely used in clinical practice (A and B), as shown in Table 1. We observed significant differences in the insertion measurements (LID and IDA) between the two evaluated electrode arrays $(p<0.05)$ because of their different design and construction characteristics.

Our study used conventional CT exams due to their widespread use for the evaluation of candidates for CI surgery. We presented practical and useful tools to evaluate CI electrodes in clinical practice, both preoperatively and postoperatively. Preoperative evaluations can help surgeons plan the surgery with regard to the $\mathrm{CI}$ electrode length, flexibility, and other features. Furthermore, surgeons can evaluate the cochlea size and possible anatomical variations. This personalized planning can benefit patients with regard to residual hearing preservation and intracochlear trauma and achieve better postoperative outcomes. Postoperative evaluations could help surgeons to evaluate the presence of possible problems during the surgery $[1,2]$. In this context, the presented tools showed to be useful to calculate insertion depth measurements, since it is easy to perform, produces high-resolution images, and is able to depict all the landmarks, thus enabling measurement of the angular and linear insertion depth of the most apical electrode contacts. Due to simplicity for implementation, the methodology can be easily applied in any center with conventional CT available.

Literature shows studies to localize CI electrodes in clinical postimplantation in CT exams [31]. A large number of image-guided CI programming methods are reported from simple manual localization of electrodes and complex computer tools such as snake-based methods [31,32] and weighted active shape model [33-36]. LID and IDA measurements may be estimated using those approaches to segment IC electrodes. Our methodology uses a free available software named ITKsnap to analyze CT images.

In conclusion, the present study evaluated two insertion depth measurements for two different CIs based on CT, a medical imaging technique that is commonly used in clinical practice. The used tools were able to comprehensively characterize Cochlear Implants A and B with regard to linear and angular depth while obviating possible variations that are caused by cochlea size. This was possible since we used the same human temporal bones for both implant models. Future studies should measure preoperative and postoperative benefits in terms of speech recognition and evaluate the preservation of residual hearing in the implanted ear. Postoperative CT imaging can also be performed to determine possible correlations between surgical factors, electrode positions, and performance. In addition to refined surgical techniques, the precise evaluation of cochlear length and correct choice of CI characteristics can play an important role in postoperative outcomes. 


\section{Conflict of Interest}

The study was developed with approval from the ethics committee of the authors' institutions and national review panels under protocol no. CAAE 41305414.0.0000.5404. The authors declare that there is no conflict of interest for this study. This research did not receive any specific grant from funding agencies in the public, commercial or notfor-profit sectors.

\section{References}

[1] Zou J, et al. Imaging cochlear implantation with round window insertion in human temporal bones and cochlear morphological variation using high-resolution cone beam CT. Acta Otolaryngol 2015;135:466-72.

[2] Whiting BR, et al. Cochlear implants: three-dimensional localization by means of coregistration of CT and conventional radiographs. Radiology 2001;221:543-9.

[3] James C, et al. Preservation of residual hearing with cochlear implantation: how and why. Acta Otolaryngol 2005;125:481-91.

[4] Gstoettner W, et al. A new electrode for residual hearing preservation in cochlear implantation: first clinical results. Acta Otolaryngol 2009;129:372-9.

[5] Skarzynski H, et al. Partial deafness treatment with the nucleus straight research array cochlear implant. Audiol Neurootol 2012;17:82-91.

[6] Carvalho GM, et al. Evaluation of the Digisonic ${ }^{\circledR}$ SP cochlear implant: patient outcomes and fixation system with titanium screws. Braz J Otorhinolaryngol 2012;78:56-62.

[7] Briggs RJ, et al. Comparison of round window and cochleostomy approaches with a prototype hearing preservation electrode. Audiol Neurootol 2006;11(Suppl. 1):42-8.

[8] Di Nardo W, et al. Residual hearing in cochlear implant patients. Eur Arch Otorhinolaryngol Aug 2007;264:855-60.

[9] Erixon E, Rask-Andersen H. How to predict cochlear length before cochlear im plantation surgery. Acta Otolaryngol 2013;133:1258-65.

[10] Skarzynski H, et al. Results of partial deafness cochlear implantation using various electrode designs. Audiol Neurootol 2009;14(Suppl. 1):39-45.

[11] Guimaraes AC, et al. Hearing preservation and cochlear implants according to inner ear approach: multicentric evaluation. Braz J Otorhinolaryngol 2015;81:190-6.

[12] Trieger A, et al. In vivo measurements of the insertion depth of cochlear implant arrays using flat-panel volume computed tomography. Otol Neurotol 2011;32:152-7.

[13] Franke-Trieger A, Murbe D. Estimation of insertion depth angle based on cochlea diameter and linear insertion depth: a prediction tool for the CI422. Eur Arch Otorhinolaryngol 2015;272:3193-9.

[14] Hod R, et al. Cochlear implantation via round window or cochleostomy: effect on hearing in an animal model. Laryngoscope Nov 2016;126:E375-8.

[15] Esquia Medina GN, et al. Is electrode-modiolus distance a prognostic factor for hearing performances after cochlear implant surgery? Audiol Neurootol 2013;18:406-13.

[16] Avci E, et al. Variations in microanatomy of the human cochlea. J Comp Neurol
2014;522:3245-61.

[17] Verbist BM, et al. Multisection CT as a valuable tool in the postoperative assessment of cochlear implant patients. Am J Neuroradiol 2005;26:424-9.

[18] Escude B, et al. The size of the cochlea and predictions of insertion depth angles for cochlear implant electrodes. Audiol Neurootol 2006;11(Suppl. 1):27-33.

[19] Whiting BR, et al. Use of computed tomography scans for cochlear implants. J Digit Imaging Sep 2008;21:323-8.

[20] Briggs RJ, et al. Development and evaluation of the modiolar research array: multicentre collaborative study in human temporal bones. Cochlear Implants Int 2011;12:129-39.

[21] Tykocinski M, et al. The contour electrode array: safety study and initial patient trials of a new perimodiolar design. Otol Neurotol 2001;22:33-41.

[22] Xu J, et al. Cochlear view: postoperative radiography for cochlear implantation. Am J Otol 2000;21:49-56.

[23] Svrakic M, et al. Measurement of Cochlear implant electrode position from intraoperative post-insertion skull radiographs: a validation study. Otol Neurotol Sep 2015;36:1486-91.

[24] Pearl MS, et al. High-resolution secondary reconstructions with the use of flat panel CT in the clinical assessment of patients with cochlear implants. AJNR Am J Neuroradiol Jun 2014;35:1202-8.

[25] Zou J, et al. X-ray microtomographic confirmation of the reliability of CBCT in identifying the scalar location of cochlear implant electrode after round window insertion. Hear Res 2015;326:59-65.

[26] Wurfel W, et al. Cochlear length determination using cone beam computed tomography in a clinical setting. Hear Res Oct 2014;316:65-72.

[27] Boyer E, et al. Scalar localization by cone-beam computed tomography of cochlear implant carriers: a comparative study between straight and periomodiolar precurved electrode arrays. Otol Neurotol Mar 2015;36:422-9.

[28] Frisch CD, et al. Evaluation of a new mid-scala cochlear implant electrode using microcomputed tomography. Laryngoscope Dec 2015;125:2778-83.

[29] Wimmer W, et al. Cone beam and micro-computed tomography validation of manual array insertion for minimally invasive cochlear implantation. Audiol Neurootol 2014;19:22-30.

[30] O'Connell BP, et al. Electrode location and angular insertion depth are predictors of Audiologic outcomes in Cochlear implantation. Otol Neurotol Sep 2016;37:1016-23.

[31] Zhao Y, et al. Automatic localization of cochlear implant electrodes in CT. Med Image Comput Comput Assist Interv 2014;17:331-8.

[32] Chakravorti S, et al. Cochlear implant phantom for evaluating computed tomography acquisition parameters. J Med Imaging (Bellingham) Oct 2017;4:045002.

[33] Noble JH, et al. Statistical shape model segmentation and frequency mapping of cochlear implant stimulation targets in CT. Med Image Comput Comput Assist Interv 2012;15:421-8.

[34] Noble JH, et al. Automatic segmentation of intracochlear anatomy in conventional CT. IEEE Trans Biomed Eng Sep 2011;58:2625-32.

[35] Wang J, et al. Retrospective evaluation of a technique for patient-customized placement of Precurved Cochlear implant electrode arrays. Otolaryngol Head Neck Surg Jul 2017;157:107-12.

[36] Reda FA, et al. An artifact-robust, shape library-based algorithm for automatic segmentation of inner ear anatomy in post-cochlear-implantation CT. Proc SPIE Int Soc Opt Eng Mar 21 2014;9034:90342V. 